\title{
Problematic alcohol and other substance use among patients presenting to emergency services in South Africa: Who is ready for change?
}

\author{
K Sorsdahl, ${ }^{1} \mathrm{PhD}$; D J Stein, ${ }^{2,3} \mathrm{MB}$ ChB, FRCPC, PhD, DPhil; T Naledi, ${ }^{4} \mathrm{MB}$ ChB, FCPHM; E Breuer, ${ }^{1} \mathrm{MPH}$; B Myers, ${ }^{2,5} \mathrm{PhD}$ \\ ${ }^{1}$ Alan J Flisher Centre for Public Mental Health, Department of Psychiatry and Mental Health, Faculty of Health Sciences, University of \\ Cape Town and Groote Schuur Hospital, Cape Town, South Africa \\ ${ }^{2}$ Department of Psychiatry and Mental Health, Faculty of Health Sciences, University of Cape Town and Groote Schuur Hospital, Cape Town, \\ South Africa \\ ${ }^{3}$ Medical Research Council Unit on Risk \& Resilience in Mental Disorders, Cape Town, South Africa \\ ${ }^{4}$ Western Cape Department of Health, Cape Town, South Africa \\ ${ }^{5}$ Alcohol and Drug Abuse Research Unit, South African Medical Research Council, Cape Town, South Africa
}

Corresponding author: K Sorsdahl (katherine.sorsdahl@uct.ac.za)

\begin{abstract}
Background. Studies that identify factors associated with intervention uptake are urgently needed in poorly resourced healthcare systems. This is important, as knowing who is likely to engage may lead to intervention targeting, which is an efficient use of scarce health resources. Objective. To identify patient characteristics that predict the acceptance of a brief intervention for substance use delivered in emergency departments (EDs).

Methods. Patients presenting to three EDs were screened for substance use using the Alcohol, Smoking, and Substance Involvement Screening Test (ASSIST). All patients identified as at risk for substance use problems were offered a brief psychotherapy intervention focused on substance user education. Data were collected on patients' age, sex, presenting condition (injury/no injury), type of substance used, and severity of substance use. Logistic regression analysis was used to identify variables that predicted acceptance of the offer of a brief intervention.

Results. Being between the ages of 25 and 39 years increased the likelihood of accepting an offer of help compared with 18 - 24-year-olds. Polysubstance users were less likely to accept an offer of help than patients with problematic alcohol use only, while patients with higher ASSIST scores were more likely to accept an offer of help than those with lower scores.

Conclusions. Findings suggest that more work is needed to understand the mechanisms underlying treatment acceptance. Brief interventions delivered in ED services in countries such as South Africa should target alcohol users with higher ASSIST scores in order to ensure the efficient use of scarce health resources.
\end{abstract}

S Afr Med J 2017;107(4):352-353. DOI:10.7196/SAMJ.2017.v107i4.10791

As close to half of the injuries treated in South African (SA) emergency departments (EDs) are substance related, ${ }^{[1]}$ these settings are important locations for identifying individuals (through screening) at risk for substance-related problems who would not normally seek treatment and for conducting brief interventions to reduce their substance use and risk for future substance-related injuries.

However, the impact of such interventions relies heavily on the willingness of patients to accept the offer of an intervention. A few studies from high-income countries have identified factors that predict intervention uptake in ED settings, but these studies have been limited to people who used alcohol. ${ }^{[2,3]}$ Studies that identify factors associated with intervention uptake are urgently needed in poorly resourced healthcare systems (such as SA's). This is important, as knowing who is likely to engage may lead to intervention targeting, which is an efficient use of scarce health resources. Furthermore, understanding who is least likely to engage may lead to strategies for enhancing intervention uptake.

\section{Objective}

To examine patient and substance use characteristics that predict the uptake of a brief intervention for substance use among patients presenting for care at EDs.

\section{Methods and results}

As part of a larger randomised controlled trial of a screening and brief intervention for substance use across four EDs in SA, ${ }^{[4]}$ a total of 2736 patients presenting for emergency services were screened for possible study inclusion. Five hundred and twenty-four nontreatment-seeking substance users ( $\geq 18$ years of age) were identified using the Alcohol, Smoking, and Substance Involvement Screening Test (ASSIST). ${ }^{[5]}$ Of these, $380(72.5 \%)$ accepted the offer of an intervention and 144 refused.

We used logistic regression analysis to examine the unadjusted and adjusted associations between intervention uptake and patients' demographic characteristics, presenting health condition and level of substance use involvement (Table 1).

In adjusted models, being between the ages of 25 and 39 years increased the likelihood (adjusted odds ratio (AOR) 1.91, 95\% confidence interval (CI) 1.02 - 3.57) of accepting an offer of help compared with substance users in the age group 18 - 24 years. Polysubstance users (AOR $0.28,95 \%$ CI $0.16-0.48$ ) were less likely to accept an offer of help than patients with problematic alcohol use only, while patients with higher ASSIST scores were more likely to accept an offer of help than those with lower scores (AOR 1.04, 95\% CI 1.00 - 1.08). 
Table 1. Unadjusted and adjusted associations between willingness to participate in a brief intervention and demographic, injury and substance use characteristics

\begin{tabular}{|c|c|c|c|c|}
\hline \multirow[b]{2}{*}{ Characteristics } & \multicolumn{2}{|c|}{ Willing to participate } & \multirow[b]{2}{*}{ Unadjusted OR (95\% CI) } & \multirow[b]{2}{*}{ Adjusted OR (95\% CI) } \\
\hline & Yes $(N=380), n(\%)$ & No $(N=144), n(\%)$ & & \\
\hline \multicolumn{5}{|l|}{ Age (yr) } \\
\hline $18-24$ & $124(32.8)$ & $43(29.9)$ & 1.00 & 1.00 \\
\hline $25-39$ & $196(51.9)$ & $74(51.4)$ & $1.34(0.76-2.38)$ & $1.91(1.02-3.57)^{*}$ \\
\hline$\geq 40$ & $58(15.3)$ & $27(18.8)$ & $1.23(0.73-2.09)$ & $1.55(0.89-2.71)$ \\
\hline \multicolumn{5}{|l|}{ Sex } \\
\hline Male & $251(66.6)$ & $103(72.0)$ & 1.00 & 1.00 \\
\hline Female & $126(33.4)$ & $40(28.0)$ & $1.29(0.85-1.97)$ & $1.00(0.63-1.57)$ \\
\hline \multicolumn{5}{|l|}{ Presented with injury } \\
\hline No & $138(36.3)$ & $47(32.6)$ & 1.00 & 1.00 \\
\hline Yes & $242(63.7)$ & $97(67.4)$ & $0.85(0.57-1.28)$ & $0.83(0.53-1.29)$ \\
\hline \multicolumn{5}{|l|}{ Substance use } \\
\hline Alcohol only & $298(78.4)$ & $85(59.0)$ & 1.00 & 1.00 \\
\hline Illicit only & $38(10.0)$ & $17(11.8)$ & $0.64(0.34-1.19)$ & $0.64(0.33-1.24)$ \\
\hline Polysubstance use & $44(11.6)$ & $42(29.2)$ & $0.30(0.18-0.49)^{*}$ & $0.25(0.15-0.42)^{*}$ \\
\hline ASSIST score, mean (SD) & $18.9(6.3)$ & $17.90(7.1)$ & $1.02(0.99-1.05)$ & $1.04(1.00-1.08)^{*}$ \\
\hline
\end{tabular}

\section{Discussion}

The findings suggest that most patients who screen positive for substance use were willing to receive an intervention to reduce their use. The findings also provide insights into how to improve patient uptake of such interventions. In contrast to previous research, presenting with a substance-related injury did not increase the likelihood of intervention uptake. ${ }^{[2]}$ This indicates that all patients seeking ED services should be screened and referred for possible substance use problems where necessary, regardless of their presenting health concern.

In contrast to patients with polysubstance use, patients with alcohol use only were more willing to receive help. The lower intervention uptake among polysubstance users and the fact that screening for polysubstance use takes longer than screening for alcohol use alone suggests that ED services should focus their efforts on addressing alcohol use in their patient population. This would be the most efficient use of scarce health resources.

Finally, although patients who screen at high risk for substance use problems in well-resourced settings are normally referred to specialist care, we contend that these patients should also be provided with a brief intervention, not only because they were the most willing to receive the intervention, but also because of limited access to specialist care in SA.

1. Pluddemann A, Parry C, Donson H, Sukhai A. Alcohol use and trauma in Cape Town, Durban and Port Elizabeth, South Africa: 1999 - 2001. Inj Control Saf Promot 2004;11(4):265-267. https://doi org/10.1080/156609704/233/289599

2. Patton R, Crawford M, Touquet R. Hazardous drinkers in the accident and emergency department who accepts advice? Emerg Med J 2004;21(4):491-492. https://doi.org/10.1136/emj.2003.011403

3. Patton R, Crawford M, Touquet R. Hazardous drinkers in the accident and emergency department who attends an appointment with the alcohol health worker? Emerg Med J 2005;22(10):722-723. https://doi.org/10.1136/emj.2004.018952

4. Sorsdahl $\mathrm{K}$, Stein DJ, Corrigall J, et al. The efficacy of a blended motivational interviewing and problem solving therapy intervention to reduce substance use among patients presenting for emergency services in South Africa: A randomized controlled trial. Subst Abuse Treat Prev Policy 2015;10:46. https://doi.org/10.1186/s13011-015-0042-1

5. World Health Organization. The Alcohol, Smoking and Substance Involvement Screening Test (ASSIST): Development, reliability and feasibility. Addiction 2002;97(9):1183-1194. https://doi. org/10.1046/j.1360-0443.2002.00185.x

Accepted 10 January 2017 